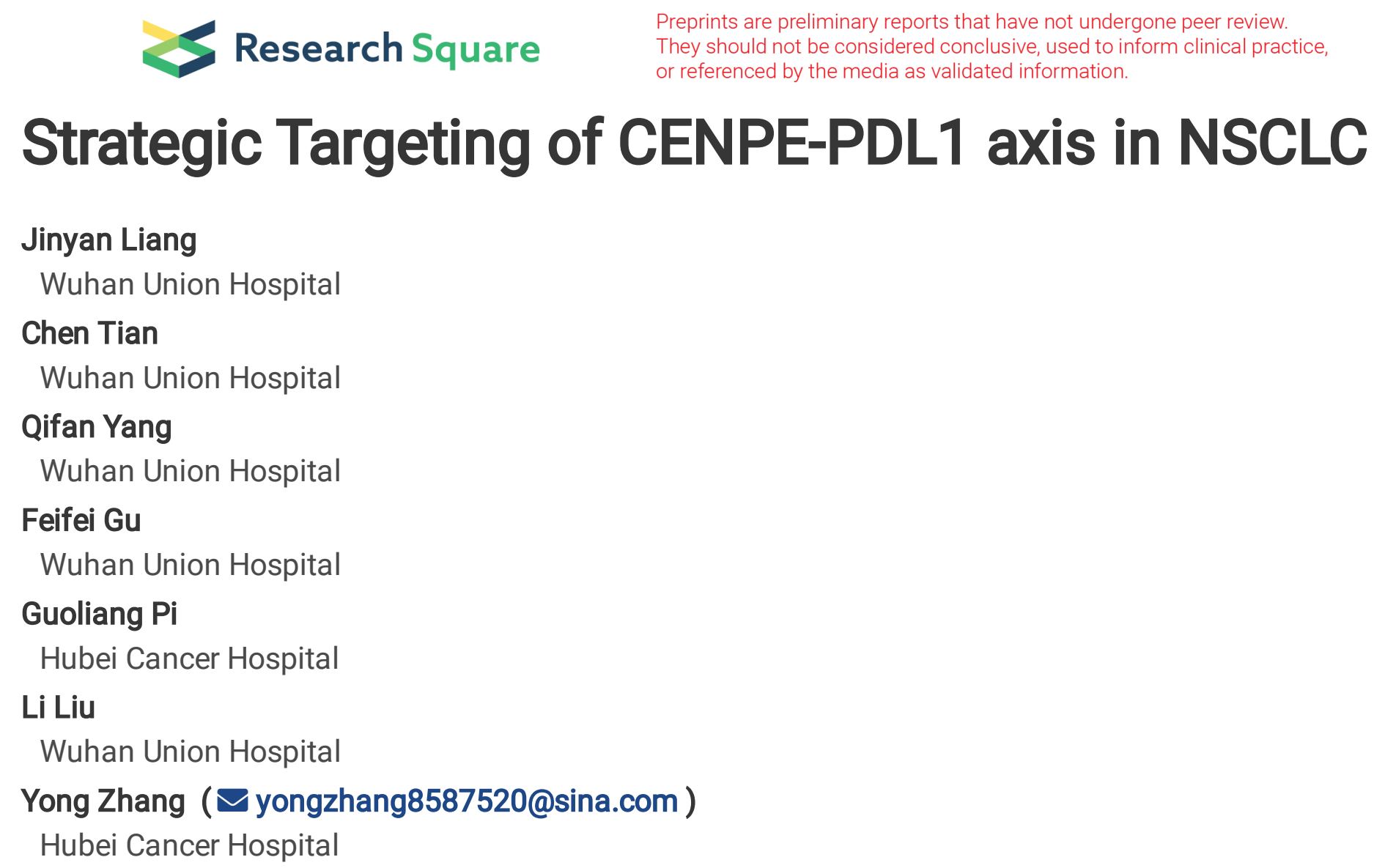

\section{Strategic Targeting of CENPE-PDL1 axis in NSCLC}

\section{Jinyan Liang}

Wuhan Union Hospital

Chen Tian

Wuhan Union Hospital

Qifan Yang

Wuhan Union Hospital

\section{Feifei Gu}

Wuhan Union Hospital

Guoliang Pi

Hubei Cancer Hospital

\section{Li Liu}

Wuhan Union Hospital

Yong Zhang ( $\square$ yongzhang8587520@sina.com )

Hubei Cancer Hospital

\title{
Research
}

Keywords: Lung cancer, CENPE inhibitor, PDL1, immunotherapy, CD8 T cells

Posted Date: November 5th, 2020

DOI: https://doi.org/10.21203/rs.3.rs-101164/v1

License: (a) This work is licensed under a Creative Commons Attribution 4.0 International License. Read Full License 


\section{Abstract}

Background Increasing evidence suggests that centromere-associated protein E (CENP-E) is expressed during mitosis and plays a key role in incorrect chromosome alignment. Therefore, CENP-E may represent a druggable target for several solid tumors.

Methods Here, we evaluated the ability of the CENPE inhibitor GSK923295 to up-regulate PDL1 and induce immune responses to tumor-associated CD8 T cell and regulatory T (Treg) cell.

Results Our study found that a CENP-E inhibitor exhibited anti-tumor activity by directly suppressing the proliferation of lung cancer cells and upregulating the expression of PD-L1. Inhibition of CENP-E suppressed antitumor immunity by attenuating the response of activated CD8+ $T$ cells and augmenting Tregs in vitro and in vivo. Mechanistically, CENP-E bound to the TTP promoter to regulate its transcription, and inhibition of CENP-E stabilized the mRNA of PD-L1 via TTP targeting of the 3'UTR. Inhibition or knockdown of CENP-E combined with an anti-PD-L1 antibody rescued the impaired antitumor CD8+ $T /$ Treg cell response and improved the antitumor effect in lung cancer. Surprisingly , further analysis found that CENP-E was only related to the poor prognosis of lung cancer.

Conclusions All of these results suggest that a CENP-E inhibitor will exert anti-tumor effects and upregulate PD-L1-induced impairment of anti-tumor CD8+/Treg cell responses in lung cancer. However, elevated PDL1 levels provide a possible strategy for combination immunotherapy, and this combination of immunotherapeutic strategies may offset the negative effects of a CENP-E inhibitor on its immunemediated antitumor ability in lung cancer treatment.

\section{Background}

Lung cancer is the most common malignant cancer in the world, and it causes the most cancer-related deaths. Global cancer statistics showed that more than 2 million new cases of lung cancer occurred in 2018 , which accounted for $11.6 \%$ of all cancers. ${ }^{1}$ According to histology, lung cancer is classified into small cell lung cancer and non-small cell lung cancer (NSCLC). NSCLC is the most common type, and it is further classified into adenocarcinoma, squamous cell carcinoma, etc. ${ }^{2}$ NSCLC is often accompanied by oncogene mutation or alteration, which firmly correlate with the development and progression of lung cancer. Current research indicates that centromere protein E (CENP-E) is related to the development and drug-resistance of lung cancer, and it is a potential driver gene in lung cancer. CENP-E is a member of the centromere-associated protein family. It plays an important role in the precise arrangement of chromosomes and cell division, and it may be a promising therapeutic target for some solid cancers, including lung cancer. ${ }^{3-7}$ CENP-E may be related with the development of familial lung cancer. ${ }^{8}$ The heterozygous knockout of CENP-E in Mad2-heterozygous knockout mice inhibited the formation of lung adenocarcinoma. ${ }^{9}$ Wood and colleagues indicated that the mRNA expression level of CENP-E was obviously elevated in lung adenocarcinoma and squamous cell carcinoma compared to normal lung tissues, and it was 5 -fold and 20 -fold higher than normal tissues, respectively. ${ }^{10}$ However, the relationship 
between the expression of CENP-E and the efficiency of immune checkpoint blockade(ICB) to lung cancer patients was not reported.

Avoidance of immune destruction and tumor promotion inflammation are newly defined characteristics of malignancies. ${ }^{11}$ Tumor cells suppress innate and acquired antitumor immunity via the downregulation $\mathrm{MHC}$ I, the upregulation of PD-L1 and the secretion of cytokines, including TGF- $\beta$ and PGE. Increasing evidence showed that traditional antitumor strategies, including targeted therapy and chemotherapy, may also play important roles in the immuno-microenvironment by regulating the expression of PD-L1. EGFRTKI restored the antitumor immunity of tumor-specific $T$ cells by suppressing the EGFR-ERK/c-jun signaling pathway and subsequently downregulating the expression of PD-L1. ${ }^{12}$ In contrast, CDK4/6 and MEK inhibitors induced PD-L1 expression. The combination of a CDK4/ 6 inhibitor or MEK inhibitor with an anti-PD-L1 antibody evidently suppressed tumor growth. ${ }^{13,14}$ However, whether the inhibition of CENPE regulates the expression of PD-L1 and the immuno-microenvironment in lung cancer is not clear.

PD-L1 is often highly expressed in cancer cells, and it is positively correlated with the invasive activity and risk of death of malignant cancer. PD-L1 in immune cells, including dendritic cells, T cells and other cells, combines with PD-1 on T cells and subsequently suppresses the proliferation of T cells, which leads to tumor immune escape. ${ }^{17}$ PD-1/PD-L1 antibodies exhibited considerable effects in lung cancer treatment. ${ }^{18}$ The objective response rate of nivolumab and pembrolumab in NSCLC was $20 \%-40 \% .{ }^{19}$ Checkpoint inhibitors were only effective in some patients, and many patients experienced primary or secondary resistance. The combination of an anti-PD-1/PD-L1 antibody and traditional therapy may be a new era in anticancer treatment. ${ }^{20}$ The combination of CENP-E inhibition with an anti-PD-L1 antibody may overcome the ICB resistance and provide a new strategy for lung cancer treatment.

In this study, we analyzed the relationship between CENP-E expression and prognosis and the expression of immune molecules in lung cancer. We further investigated the influence of CENP-E inhibition on PD-L1 expression in lung cancer cells and antitumor immunity. Finally, the antitumor efficacy of the inhibition of CENP-E combined with an anti-PD-L1 antibody in lung cancer was examined.

\section{Materials And Methods}

\section{Cell lines and cell culture}

NSCLC cell lines A549, H522 and Lewis were purchased from the Shanghai Institute of Cell Biology (Shanghai, China). A549 and H522 were cultured with RPMI-1640 complete medium supplemented with $10 \%$ fetal bovine serum and antibiotics $(100 \mathrm{U} / \mathrm{ml}$ streptomycin and $100 \mathrm{U} / \mathrm{ml}$ penicillin). Murine lung cancer cell line Lewis was propagated in DMEM complete medium. Human mononuclear cells derived from malignant pleural effusion (MPE) of lung cancer patients were grown in IMDM, and the medium was supplemented with $50 \mathrm{mM}$ 2-mercaptoethanol. 


\section{Co-culture system of A549 and monocuclear cells derived from MPE}

To analyse the effect of CENP-E inhibition in A549 on immunity, A549 treated with GSK923295 for 3 days was co-cultured with monocuclear cells isolated from MPE. Co-cultures at 1:100 (tumor cell: monocuclear cell) ratio were incubated in the presence of anti-CD3/CD28 antibody (Thermo, $1 \mathrm{ug} / \mathrm{ml}$ ) and IL-2 (PeproTech, $100 \mathrm{U} / \mathrm{ml}$ ) for 4 days. Then the monocuclear cells were collected for the FACS analyses. For the colony formation assays to investigate the combinatorial antitumor effect with GSK923295 and PDL1 antibody Atezolizumab (Selleckchem, 10ug/ml), A549 treated with GSK923295 for 3 days was cocultured with monocuclear cells at the ratio of 1:12, 1:25, 1:50 and 1:100. About 10 days later, the tumor cells on the plates were fixed with $4 \%$ paraformaldehyde and washed with PBS, then was stained with $0.5 \%$ crystal violet solution.

\section{FACS analyses}

To perform FACS analyses, single-cell suspensions were prepared in PBS with $2 \%$ BSA. For the detection of PD-L1 and MHC I in A549 and H522, the suspension cells were stained with PD-L1-PE Cy7 and MHC IAPC (ebioscience). For the evaluation of $\mathrm{CD}^{+}{ }^{+} \mathrm{T}$ cells and Tregs, the cells were stained with the following anti-human/mouse antibodies targeting surface markers at $4{ }^{\circ} \mathrm{C}$ for 30 minutes: CD8-FITC, 7-AAD, CD4FITC, CD25-PE, CD107-PE and Foxp3-PE (ebioscience, Biolegend). The expression of IFN-y and Foxp3

were detected by using BD Cytofix/Cytoperm kit (BD Biosciences) according to the manufacturer's instructions. The stained cells were analyzed using a FACSAria II flow cytometer (BD Biosciences). The data was analyzed with FlowJo software.

\section{Animal studies}

Female C57BL/ 6 mice of 6-8 weeks old were purchased from Liaoning Changsheng Biotechnology Company. The 6-weeks old nude female mice were obtained from Beijing Vital River Laboratory Animal Technology Company. All procedures on mice were conducted according to the guidelines of the Animal Care and Use Committee at Huazhong University of Science and Technology.

To study the therapeutic effect of GSK923295 in A549 nude mice models, A549 cells ( $1 \times 10^{7}$ cells) were suspended in $50 \mu \mathrm{l}$ of RPMI- 1640 mixed with $50 \mu$ of Matrigel Basement Membrane Matrix (BD Biosciences) and injected subcutaneously into 6-week female nude mice. When tumor volume reached $200 \mathrm{~mm}^{3}$, mice were randomly divided into two groups with comparable average tumor size (5 mice per group). Mice were then treated with vehicle alone or GSK923295 (125 mg/kg; Selleckchem) intraperitoneally in two cycles of three daily injections.

To study the immunoregulatory effect of CENP-E inhibition and the combinatorial antitumor effect with CENP-E inhibition and PD-L1 antibody, the mice were randomized into three groups (5 mice per group in the study of immunoregulatory effect of CENP-E inhibition and 10 mice per group in the study of combinatorial treatment). Lewis lung tumor mice model were established by subcutaneously injecting 1 
$\times 10^{6}$ control- or CENP-E knockdown- Lewis cells in $100 \mu$ DMEM into the right flank of mouse. To characterize the tumor-infiltrating immune cells, the mice were sacrificed after 14 days. Then the subcutaneous tumors were collected for FACS analyses. For PD-L1 antibody treatment, 10 mice in each group were divided into two groups with comparable average tumor size ( 5 mice per group), then $200 \mu \mathrm{g}$ of PD-L1 antibody (Bio X Cell, 10F.9G2) or control IgG (Bio X Cell, LTF-2) was injected intraperitoneally every three days for a total of 8 injections.

For survival studies, tumor models were established and treated with the same method described above. The mice were monitored for tumor volumes every three days for 90 days after initial implantation. Animals whose tumors exceeded $2,000 \mathrm{~mm}^{3}$ were euthanized.

Tumor sizes were measured by callipers and the tumor volume was calculated using the formula: $W^{2} \times L$ $\times 0.5$, where $L$ is the longest dimension and $W$ is the corresponding perpendicular dimension.

\section{Patients and Clinical samples}

The malignant pleural effusion (MPE) and lung tumor tissues were collected from lung cancer patients in the Cancer Center, Union Hospital, Tongji Medical College of Huazhong University of Science and Technology. These effusions were histologically diagnosed as malignant pleural effusion, and will be utilized in co-culture system with tumor cells. The lung tumor tissues were collected for immunofuorescence or immunohistochemical staining to investigate the expression of CENP-E and PDL1. This study was approved by the Cancer Center of Union Hospital in Wuhan, P.R.China. Informed consents were obtained from these patients.

\section{RNA sequencing}

After A549 was treated with $100 \mathrm{nmol} / \mathrm{L}$ GSK923295, cells were washed with PBS and collected for RNA extraction. The RNA was amplified to build up the single-stranded circular DNA bank. BGISEQ-500 platform was utilized for the subsequent sequencing.

\section{Statistics analyses}

All analyses were conducted by using GraphPad Prism software. All assays were conducted for at least three times. Data were presented as the mean \pm SD. Student's $t$ test was performed to compare two groups of independent samples. Kaplan-Meier curves and log-rank tests were used to evaluate the statistical differences between groups in survival studies. $P$ values less than 0.05 were considered statistically significant.

\section{Results}

\section{The role of CENP-E inhibitors in lung cancer cells}

To demonstrate the effect of CENP-E on A549 proliferation, colony formation and the cell cycle, we inhibited CENP-E using the CENP-E-specific inhibitor GSK923295. The IC50 of GSK923295 at $72 \mathrm{~h}$ was 
$43.79 \mathrm{nmol} / \mathrm{L}$ (Fig. 1A). Therefore, we treated A549 and H522 cells with GSK923295 for the CCK8 assays, colony formation assays and PI staining. Our data showed that CENP-E inhibition with GSK923295 inhibited the proliferation of A549 and H522 cells, especially at $72 \mathrm{~h}$ and at 50 and $100 \mathrm{nmol} / \mathrm{L}$ (Fig. 1BC). Significantly decreased colony formation numbers were observed in A549 and H522 cells treated with GSK923295 (Fig. 1D). The inhibition of CENP-E arrested the cell cycle of A549 and H522 cells at the G2/M phase (Fig. 1E). Therefore, our data indicated that CENP-E inhibition exhibited anti-tumor activity by directly suppressing the proliferation of tumor cells and arresting the cell cycle.

CENP-E inhibition upregulates PDL1 expression in lung cancer cells.

To elucidate the influence of CENP-E inhibition on immunity, we conducted RNA sequencing. We investigated the expression of a total of 17135 genes and found that 429 genes were upregulated and 331 genes were downregulated in A 549 cells treated with GSK923295 (Fig. 2A). We further investigated the distinctive genes related with immunity and PD-L1. We found that PD-L1 was obviously highly expressed after CENP-E inhibition. The expression of immunosuppressive molecules, including $I L 1 \beta, I L-6$, $I L-33$ and $P T G S 2$, were also elevated, and genes that participate in the regulation of PD-L1, including IFNGR, CXCL8, IL-6, JAK3 and JUN, were upregulated (Fig. 2B).

To verify the influence of CENP-E on PD-L1 expression in lung cancer, we evaluated the expression of PDL1 in A549 and H522 cells treated with the CENP-E-specific inhibitor GSK923295. Our data showed that GSK923295 induced PD-L1 expression at the mRNA and protein level at 50 and $100 \mathrm{nmol} / \mathrm{L}$ (Fig. 2C and D). GSK923295 also increased the expression of MHC I in lung cancer cells at 50 and $100 \mathrm{nmol} / \mathrm{L}$ (Fig. 2E). We further confirmed the expression of CENP-E and PD-L1 in lung cancer tissues from patients. The results indicated a negative correlation between CENP-E and PD-L1 in lung cancer tissues (Fig. 2F). Therefore, the inhibition of CENP-E in lung cancer induced the expression of PD-L1 at the mRNA and protein level.

CENP-E signal inhibition induces T-cell suppression in vitro and in vivo.

To explore whether the CENP-E inhibition-induced upregulation of PD-L1 in A549 cells affected antitumor immunity, we cultured mononuclear cells isolated from MPE and A549 cells treated with GSK923295. A549 cells treated with GSK923295 exhibited a dose-dependent reduction in the ratio of $\mathrm{CD}^{+} \mathrm{T}$ cells to $\mathrm{CD} 4^{+} \mathrm{T}$ cells and an increase in the proportion of apoptotic $\mathrm{CD} 8^{+} \mathrm{T}$ cells $\left(7-\mathrm{AAD}^{+} \mathrm{CD} 8^{+}\right)$and apoptotic $\mathrm{CD}^{+} \mathrm{T}$ cells $\left(7-\mathrm{AAD}^{+} \mathrm{CD} 4^{+}\right)$(Fig. $3 \mathrm{~A}$ and $B$ ). We also found that $\mathrm{A} 549$ cells treated with 50 and $100 \mathrm{nmol} / \mathrm{L}$ GSK923295 resulted in the elevation of Tregs (Fig. 3C).

To verify the in vivo influence of CENP-E on lung tumor growth, an A549 nude mouse model was established and treated with GSK923295. GSK923295 significantly suppressed A549 growth in nude mice and prolonged the survival of tumor-bearing nude mice (Fig. 3D). We further established a CENP-E knockdown Lewis cells line and a subcutaneous Lewis lung cancer C57BL/6 mice model. Our results indicated that the knockdown of CENP-E slightly suppressed tumor growth in Lewis lung tumor-bearing C57BL/ 6 mice, but it did not affect the survival of immunocompetent tumor bearing mice (Fig. 3E and $F$ ). 
CENP-E knockdown reduced the infiltration of $\mathrm{CD}^{+} \mathrm{T}$ cells and the secretion of IFN-y in $\mathrm{CD} 8^{+} \mathrm{T}$ cells and increased the proportion of Tregs compared to control mice (Fig. 3G). Therefore, the inhibition of CENP-E in lung cancer reduced $\mathrm{CD} 8^{+} \mathrm{T}$ cells and elevated Tregs, which led to immunosuppression.

The inhibition of CENP-E stabilized PD-L1 mRNA by TTP targeting of the 3'UTR.

To determine whether CENP-E regulated the stability of PD-L1 mRNA and its mechanism, we conducted mRNA stability assays. The inhibition or knockdown of CENP-E retarded the degradation of PD-L1 mRNA and enhanced the stability of PD-L1 mRNA (Fig. 4A). A luciferase reporter gene assay was performed to further investigate whether the 3' UTR mediated this influence on the stability of PD-L1 mRNA. The inhibition of CENP-E using GSK923295 in PD-L1 3'UTR wild-type A549 cells enhanced the luciferase activity and stabilized the mRNA. After mutation in the PD-L1 3'UTR, the inhibition of CENP-E did not increase the luciferase activity (Fig. 4B). Therefore, the influence of CENP-E on the stability of PD-L1 mRNA was mediated by the PD-L1 3'UTR. To identify the proteins regulating the stability of PD-L1 mRNA, we performed a selected screen of likely candidate genes TTP and UPF1 (both genes regulate mRNA stability via the 3'UTR). PD-L1 expression was increased after the knockdown of TTP in A549 cells, not UPF1 knockdown (Fig. 4C). The inhibition of CENP-E in A549 cells downregulated TTP expression, and the high expression of TTP inhibited PD-L1 expression. The CENPE inhibitor-induced upregulation of PDL1 was rescued with the upregulation of TTP (Fig. 4D). The CENP-E protein was bound to the TTP promoter (Fig. 4E). We used a TTP primary antibody to immunoprecipitate the bound RNA. The results demonstrated considerable mRNA expression of PD-L1 in IP samples, which indicates the interaction between TTP and the mRNA of PD-L1 (Fig. 4F). Therefore, CENP-E stabilized PD-L1 mRNA via the 3'UTR, which was mediated by TTP. All of these findings show that CENP-E bound and induced of the transcription of TTP, and the inhibition of CENP-E stabilized PD-L1 mRNA by TTP targeting of the 3'UTR.

Synergy between CENP-E inhibition and PD-L1 mAbs enhanced the antitumor effect.

Our previous data demonstrated that GSK923295 induced PD-L1 expression in A549 cells and resulted in immunosuppression. This pathway may be the mechanism for future resistance, and the high expression of PD-L1 may indicate that the patient would benefit from an anti-PD-L1 antibody. We cultured A549 cells with mononuclear cells isolated from non-small cell lung cancer patient MPE to investigate the efficacy of the combination therapy of GSK923295 and an anti-PD-L1 antibody atezolizumab. The results showed no difference between A549 cells treated with an anti-PD-L1 antibody and no treatment. GSk923295 slightly suppressed the colony formation ability of A549 cells, and the treatment of GSK923295 combined with anti-PD-L1 antibody inhibited colony formation activity more efficiently (Fig. 5A). Control and CENP-E knockdown Lewis lung cancer-bearing mice were treated with PD-L1 antibodies. CENP-E knockdown without anti-PD-L1 antibody treatment and PD-L1 treatment alone in control mice suppressed tumor growth to some extent, and anti-PD-L1 antibody treatment in CENP-E knockdown mice inhibited the tumor growth more obviously (Fig. $5 \mathrm{C}$ and $D$ ). Survival was also significantly improved by the combination treatment of PD-L1 antibodies with CENP-E knockdown (Fig. 5E). Tumors treated with the 
anti-PD-L1 antibody enhanced the effector CD8 + T cell response, and the tumor-infiltrating Treg response was impaired. (Fig. 5F and G).

High expression of CENP-E indicated poor prognosis in lung cancer.

We analyzed the expression of CENP-E in lung cancer using the website UALCAN and found obvious high expression of CENP-E in lung adenocarcinoma and squamous cell carcinoma. The expression of CENP-E was 5.8 -fold higher in lung adenocarcinoma tissues than normal tissues $(0.304$ vs. $2.073, p<0.0001)$. The expression of CENP-E was 13.5-fold higher in lung squamous cell carcinoma tissues than normal tissues ( 0.385 vs. $5.571, p<0.0001$ ) (Fig. $6 \mathrm{~A}$ ). We used the TCGA database to analyze the expression levels of various tumor CENP-E proteins in patients. We found high expression of CENP-E in lung cancer, colon cancer, breast cancer, stomach cancer, urothelial cancer, testicular cancer and melanoma (Fig. 6B). However, further analysis found that CENP-E was only related to the prognosis of lung cancer (Fig. S1).

To explore the prognostic significance of CENP-E in lung cancer patients, bioinformatic analysis was conducted using the website Kaplan-Meier Plotter. Lung cancer patients with high CENP-E expression had better outcome than patients with low CENP-E expression, including FP (first progression) (10 months vs. 28 months, $p<0.0001$ ), OS (overall survival) (45 months vs. 96 months, $p<0.0001$ ) and PPS (post progression survival) (10.6 months vs. 19.47 months, $p=0.012$ ) (Fig. $6 \mathrm{C}$ ). Collectively, all of the TCGA data suggested the prognostic value of CENP-E in lung adenocarcinoma patients.

Bioinformatic analysis was performed using the website R2: Genomics Analysis and Visualization Platform to explore the relationship between the expression of CENP-E and immune molecules. Surprisingly, the results indicated that CENP-E in lung cancer tissues negatively correlated with checkpoint PD-L1 (CD274), the ligand of TIM3 galectin-9 and the ligand of BTLA HVEM. CENP-E in lung cancer tissues positively correlated with the immunostimulating cytokines IL2 and IL12B and negatively correlated with the immunosuppressive factor TGFB (Fig. 6D). Therefore, our results demonstrated the relationship between CENP-E and the outcome of patients and the expression of immune checkpoint and cytokines in lung cancer. All of these results suggest that a CENP-E inhibitor would exhibit anti-tumor effects and upregulate PD-L1-induced immunosuppression in lung cancer. Elevated PD-L1 levels provide a possible strategy for combination immunotherapy, and this combination of immunotherapeutic strategies may offset the negative effects of a CENP-E inhibitor on its immune-mediated antitumor abilities in lung cancer treatment.

\section{Discussion}

Our study demonstrated the expression and prognostic significance of CENP-E in lung cancer. The inhibition of CENP-E stabilized PD-L1 mRNA via TTP targeting of the 3'UTR and subsequently induced PD-L1 expression, which inhibited the antitumor immunity. The combination of GSK923295 and an antiPD-L1 antibody improved the antitumor efficacy. Our data determined the effect of CENP-E in the lung cancer immunomicroenvironment, and provided evidence for the use of combination therapy of GSK923295 and checkpoint inhibitors, including an anti-PD-L1 antibody, in NSCLC. 
According to our bioinformatic analysis, the expression of CENP-E was upregulated in lung adenocarcinoma and squamous cell carcinoma and negatively correlated with the prognosis of lung cancer patients. These results are consistent with other previously reported research. Tomoshige and colleagues indicated that CENP-E somatic mutation was related with familial lung cancer. ${ }^{8}$ Researchers also reported the high expression of CENP-E in lung adenocarcinoma and squamous cell carcinoma compared to that in normal tissues. ${ }^{10}$ Our research is consistent with these previous results. Our analysis was based on the latest data from TCGA and enrolled more cases. In addition to the expression of CENP$E$ in lung cancer, we analyzed the relationship between CENP-E expression and patient prognosis, including short-term prognosis first progression (FP) and long-term prognosis overall survival (OS). We also analyzed the relationship between the expression of CENP-E and immune molecules, which was not reported previously. Our preliminary research on the relationship between CENP-E and immune molecule expression suggested a link between CENP-E and the immunomicroenvironment, which lays the foundation for the influence of CENP-E in antitumor immunity.

Genetic alterations in lung cancer cells may be closely related with the lung cancer microenvironment. Antitumor treatment, including chemotherapy and targeted therapy, may also result in immunosuppression and resistance. The immunoregulatory function of CENP-E in malignancies and other diseases was not reported. We speculated that CENP-E regulated antitumor immunity via PD-L1. Our previous bioinformatic analysis on the relationship between CENP-E and immune molecule expression demonstrated a link between CENP-E and the immuno-microenvironment. The present study is the first study to investigate the impact of CENP-E on PD-L1 expression. The inhibition of CENP-E induced the expression of PD-L1 in lung cancer cells. This inhibition may result in PD-LI-mediated immunosuppression and lead to the recurrence and progression of lung cancer.

Inflammatory factor signaling pathways, oncogene signaling pathways, posttranscriptional regulation, and microRNAs participate in the regulation of PD-L1. ${ }^{21-24}$ According to the upregulation of PD-L1 expression at the mRNA and protein level, CENP-E may regulate PD-L1 via a transcriptional or posttranscriptional manner. Our research revealed that the inhibition of CENP-E stabilized PD-L1 mRNA in the $3^{\prime} U T R$ targeted by TTP. The regulation of the 3'UTR for PD-L1 expression and the function of TTP in the stability of PD-L1 mRNA were reported in lymphoma and gastric cancer. ${ }^{25,26}$ However, their role in PDL1 mRNA in lung cancer was not known. Our study presented the importance of the 3'UTR targeted by TTP for the stability of PD-L1 mRNA in lung cancer.

The binding of PD-L1 with PD-1 inhibits the immune activity of T cells, suppresses the activation and proliferation of $\mathrm{T}$ cells and leads to the apoptosis of T cells. ${ }^{18}$ Our research treated A549 cells with GSK923295, which reduced the ratio of $C D 8^{+} T$ to $C D 4^{+} T$ cells and induced the apoptosis of $C D 8^{+} T$ and $\mathrm{CD}^{+} \mathrm{T}$ cells, especially $\mathrm{CD} 8^{+} \mathrm{T}$ cells. This result indicated that the GSK923295-induced high expression of PD-L1 decreased the ratio of $\mathrm{CD} 8^{+} \mathrm{T}$ to $\mathrm{CD} 4^{+} \mathrm{T}$ cells, likely via the induction of $\mathrm{T}$ cell apoptosis. The binding of PD-L1 with PD-1 also suppresses immunity via other immune cells. PD-L1 on the surface of dendritic cells stimulates the differentiation of Foxp $3^{+} \mathrm{T}$ cells. The knockdown of PD- 1 in $\mathrm{CD} 4^{+} \mathrm{T}$ cells 
inhibits their differentiation into peripheral Tregs, which indicates the significant value of the PD-L1/PD-1 pathway in the differentiation of peripheral Tregs. ${ }^{27}$ Consistent with their research, our data demonstrated that the high expression of PD-L1 in A549 cells elevated the proportion of Tregs, which indicates the importance of PD-L1/PD-1 for the maintenance of Tregs. Wood's study verified that GSK923295 suppressed the tumor growth in an A549 lung cancer nude mice model. ${ }^{28}$ To investigate the influence of CENP-E on the lung cancer microenvironment, we also established a Lewis lung cancer model in immuneintact C57 mice. Similar to Wood's research, we also found that the knockdown of CENP-E suppressed tumor growth and decreased the infiltration of $\mathrm{CD}^{+} \mathrm{T}$ cells and its secretion of IFN- $\gamma$, accompanied with an elevation of Tregs. These effects on immunity were totally unexplored previously.

Checkpoint inhibitors have made great progress in NSCLC treatment. However, primary and secondary resistance restrict their application in lung cancer. ${ }^{19}$ The combination of checkpoint inhibitors and other treatment strategies, such as radiotherapy, chemotherapy, targeted therapy and other immunotherapy, may be a solution. ${ }^{20}$ Sheng and Luo suggested that patient with an upregulation of MHC I and PD-L1 in cancer cells may benefit from PD-1/PD-L1 antibodies. ${ }^{15,29}$ In our study, the increase in PD-L1 expression in A549 cells induced by the CENP-E-specific inhibitor GSK923295 altered immune cells, including T cells and Tregs, and led to immunosuppression. The inhibition of CENP-E also elevated the expression of MHC I. These data indicated better treatment efficacy of GSK923295 when combined with an anti-PD-1/PD-L1 antibody. Our further in vitro and in vivo experiments verified our speculation. We explored the combination treatment of CENP-E inhibition or knockdown using an anti-PD-L1 antibody in lung cancer for the first time. Our data provide evidence for the use of the CENP-E-specific inhibitor GSK923295 as a single agent or in combination with immunotherapy, including checkpoint inhibitors, in NSCLC.

\section{Conclusions}

Our research was the first study to explore the immuno-regulatory function of CENP-E inhibition in lung cancer, and we demonstrated that the combination of CENP-E inhibition with an anti-PD-L1 antibody in lung cancer improved antitumor efficiency. PD-L1 upregulation is a biomarker for the efficacy of anti-PD1 and anti-PD-L1 combination therapies, ${ }^{30}$ and our study provides a safe and effective strategy to overcome CENP-E inhibitor resistance with the combination of anti-PD1 or anti-PD-L1 therapy. The encouraging preclinical data warrants further testing of this strategy in clinical trials for NSCLC. ${ }^{31}$

\section{Abbreviations}

\section{CENPE}

centromere protein $\mathrm{E}$

NSCLC

Non-small cell lung cancer

Treg

regulated $\mathrm{T}$ cell 


\section{EGFR-TKI}

epidermal growth factor receptor (EGFR) kinase inhibitors

OS

Overall survival

PBS

Phosphate-buffered saline

qRT-PCR

RNA extraction and quantitative polymerase chain reaction

\section{RNA-Seq}

RNA sequencing

RIP

RNA immunoprecipitation

TCGA

The Cancer Genome Atlas

\section{Declarations}

\section{Author contributions}

J.L., and Y.Z designed and performed the experiments. C.T ,G.P and Q. Y. performed the experiments. F. G. performed the bioinformatics analysis. Y. L., Y.Z, Y.L performed some experiments. Y.H and K.Z provided essential tools. L.L and Y. Z designed and supervised the project.

\section{Availability of data and materials}

The original data will be transferred and shared following the instruction by the Editorial Committee.

\section{Acknowledgement}

We appreciate Shenzhen Huada Gene Technology Co Ltd for RNA seq.

\section{Funding:}

This work was supported by Three grants from the National Natural Science Foundations of China (No. 81773056,81602501 ) and one grant from the Natural Science Foundations of Hubei Province (No. 2016CFB217). The $7^{\text {th }}$ Wuhan Young and

Middle-aged Backed Talent of Medical Trianing Project(2019No.87)

\section{Author Information}

Jinyan Liang, Chen Tian, Qifan Yang, Feifei Gu and Guoliang Pi contributed equally to this work. 
The study was endorsed by the Research Ethics Committee of Huazhong University of Science and Technology . Informed consents were obtained from all participating patients. This study complied with the Animal Care guidelines of Huazhong University of Science and Technology.

\section{Consent for publication}

Informed consent for publication was obtained from all participants

\section{Competing interests}

No conflicts of interest were disclosed.

\section{References}

1. Bray F, Ferlay J, Soerjomataram I, et al. Global cancer statistics 2018: GLOBOCAN estimates of incidence and mortality worldwide for 36 cancers in 185 countries. CA Cancer J Clin. 2018; 68:394424.

2. Petersen I, Warth A. Lung cancer: developments, concepts, and specific aspects of the new WHO classification. J Cancer Res Clin Oncol. 2016;142:895-904.

3. Ohashi $A$, Ohori $M$, Iwai $K$, et al. Aneuploidy generates proteotoxic stress and DNA damage concurrently with p53-mediated post-mitotic apoptosis in AC-impaired cells. Nat Commun. 2015; 6:7668.

4. Hou S, Li N, Zhang Q, et al. XAB2 functions in mitotic cell cycle progression via transcriptional regulation of CENPE. Cell Death Dis. 2016; 7:e2409.

5. Schaar BT, Chan GKT, Maddox P, et al. CENP-E Function at Kinetochores Is Essential for Chromosome Alignment. J Cell Biol. 2002; 139: 1373-1382.

6. Sardar HS, Luczak VG, Lopez MM, et al. Mitotic Kinesin CENP-E Promotes Microtubule Plus-End Elongation. Curr Biol. 2011;20:1648-1653.

7. Ohashi A, Ohori M, Iwai K. Motor activity of centromere-associated protein-E contributes to its localization at the center of the midbody to regulate cytokinetic abscission. Oncotarget. 2016;7:79964-79980.

8. Tomoshige K, Matsumoto K, Tsuchiya T, et al. Germline mutations causing familial lung cancer. $J$ Hum Genet. 2015;60:597-603.

9. Silk $A D$, Zasadil LM, Holland AJ, et al. Chromosome missegregation rate predicts whether aneuploidy will promote or suppress tumors. Proc Natl Acad Sci. 2013; 110: E4134-E4141.

10. Wood KW, Chua P, Sutton D, et al. Centromere-associated protein E: A motor that puts the brakes on the mitotic checkpoint. Clin Cancer Res. 2008;14:7588-7592.

11. Hanahan D, Weinberg RA. Hallmarks of cancer: The next generation. Cell. 2011;144:646-674.

12. Chen N, Fang W, Zhan J, et al. Upregulation of PD-L1 by EGFR activation mediates the immune escape in EGFR-driven NSCLC: Implication for optional immune targeted therapy for NSCLC patients 
with EGFR mutation. J Thorac Oncol. 2015;10:910-923.

13. Zhang J, Bu X, Wang H, et al. Cyclin D-CDK4 kinase destabilizes PD-L1 via cullin 3-SPOP to control cancer immune surveillance. Nature. 2018;553:91-95.

14. Kang SH, Keam B, Ahn YO, et al. Inhibition of MEK with trametinib enhances the efficacy of anti-PDL1 inhibitor by regulating anti-tumor immunity in head and neck squamous cell carcinoma. Oncoimmunology. 2019;8:1-11.

15. Sheng W, LaFleur MW, Nguyen TH, et al. LSD1 Ablation Stimulates Anti-tumor Immunity and Enables Checkpoint Blockade. Cell. 2018;174:549-563.

16. Liang Y, Ahmed M, Guo H, et al. LSD1-mediated epigenetic reprogramming drives CENPE expression and prostate cancer progression. Cancer Res. 2017;77:5479-5490.

17. Dong H, Strome SE, Salomao DR, et al. Tumor-associated B7-H1 promotes T-cell apoptosis: A potential mechanism of immune evasion. Nat Med. 2002;8:793.

18. Xu-Monette ZY, Zhang M, Li J, et al. PD-1/PD-L1 blockade: Have we found the key to unleash the antitumor immune response? Front Immunol. 2017;8:1597.

19. Pitt JM, Vétizou M, Daillère R, et al. Resistance Mechanisms to Immune- Checkpoint Blockade in in Cancer: Tumor-Intrinsic and -Extrinsic Factors. Immunity. 2016; 44: 1255-1269.

20. Wargo JA, Cooper ZA, Flaherty KT. Universes collide: Combining immunotherapy with targeted therapy for cancer. Cancer Discov. 2014; 4: 1377-1386.

21. Zhang J, Dang F, Ren J, et al. Biochemical Aspects of PD-L1 Regulation in Cancer Immunotherapy. Trends Biochem Sci. 2018; 43:1014-1032.

22. Sun C, Mezzadra R, Schumacher TN. Regulation and Function of the PD-L1 Checkpoint. Immunity. 2018; 48:434-452.

23. Li CW, Lim SO, Xia W, et al. Glycosylation and stabilization of programmed death ligand-1 suppresses T-cell activity. Nat Commun. 2016;7:12632.

24. Hsu JM, Xia W, Hsu YH, et al. STT3-dependent PD-L1 accumulation on cancer stem cells promotes immune evasion. Nat Commun. 2018; 9:1908.

25. Kataoka K, Shiraishi Y, Takeda Y, et al. Aberrant PD-L1 expression through 3'-UTR disruption in multiple cancers. Nature. 2016;534: 402.

26. Guo J, Qu H, Shan T, et al. Tristetraprolin highecpression in gastric cancer cells suppresses PD-L1 expression and inhibits tumor progression by enhancing antitumor immunity. Mol Cells. 2018;41: 653-664.

27. Chen X, Fosco D, Kline DE, et al. PD-1 regulates extrathymic regulatory T-cell differentiation. Eur J Immunol. 2014;44:2603-2616.

28. Wood KW, Lad L, Luo L, et al. Antitumor activity of an allosteric inhibitor of centromere-associated protein-E. Proc Natl Acad Sci. 2010; 107: 5839-5844.

29. Luo N, Nixon MJ, Gonzalez-Ericsson PI, et al. DNA methyltransferase inhibition upregulates MHC-I to potentiate cytotoxic T lymphocyte responses in breast cancer. Nat Commun. 2018;9:1-11. 
30. Reck M, Rodríguez-Abreu D, Robinson AG, et al. Pembrolizumab versus chemotherapy for PD-L1positive non-small-cell lung cancer. N Engl J Med. 2016;375:1823-33.

31. Chung V, Heath El, Schelman WR, et al. First-time-in-human study of GSK923295, a novel antimitotic inhibitor of centromere-associated protein e (CENP-E), in patients with refractory cancer. Cancer Chemother Pharmacol. 2012; 69(3):733-741.

\section{Figures}


A

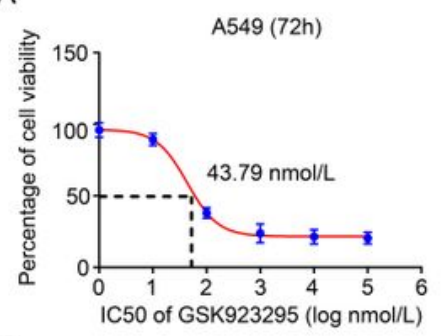

C
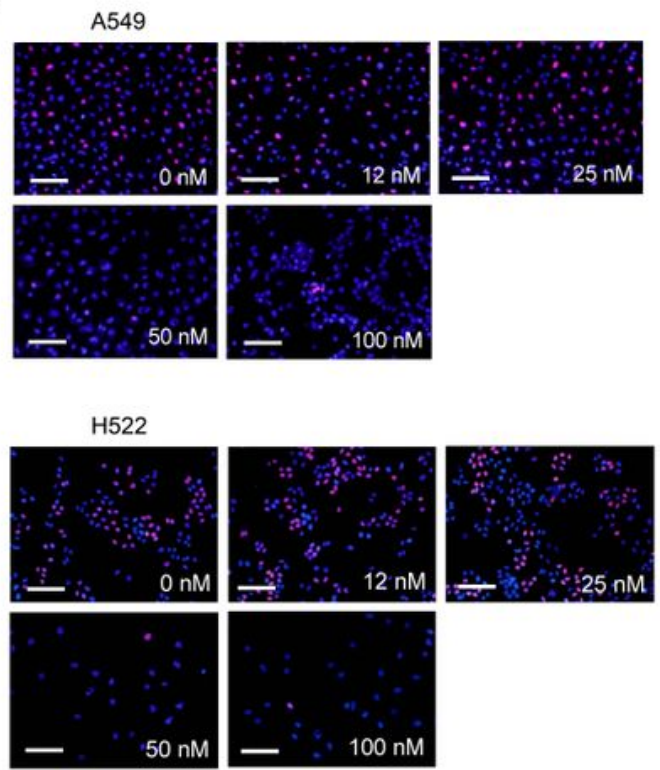

E ${ }_{\text {A549 }}$
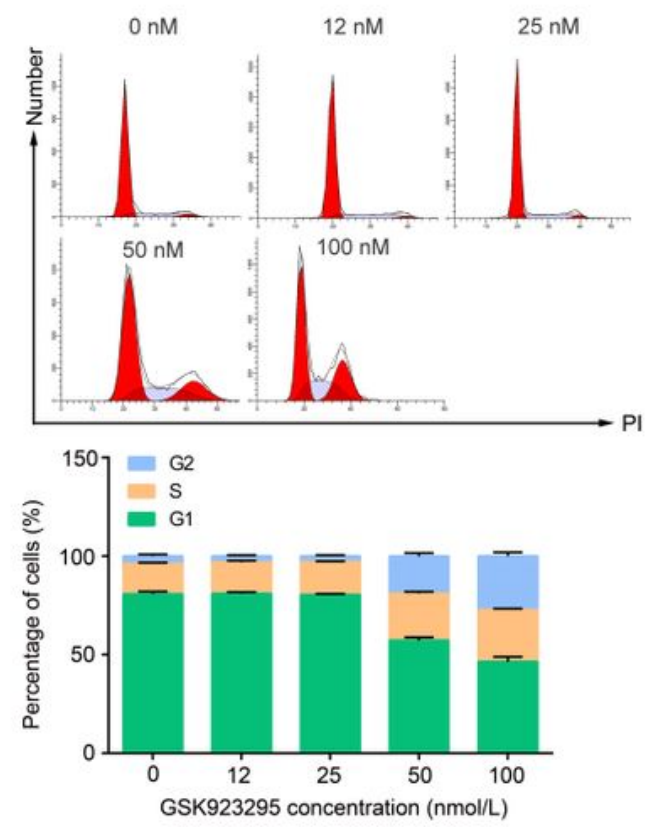

D
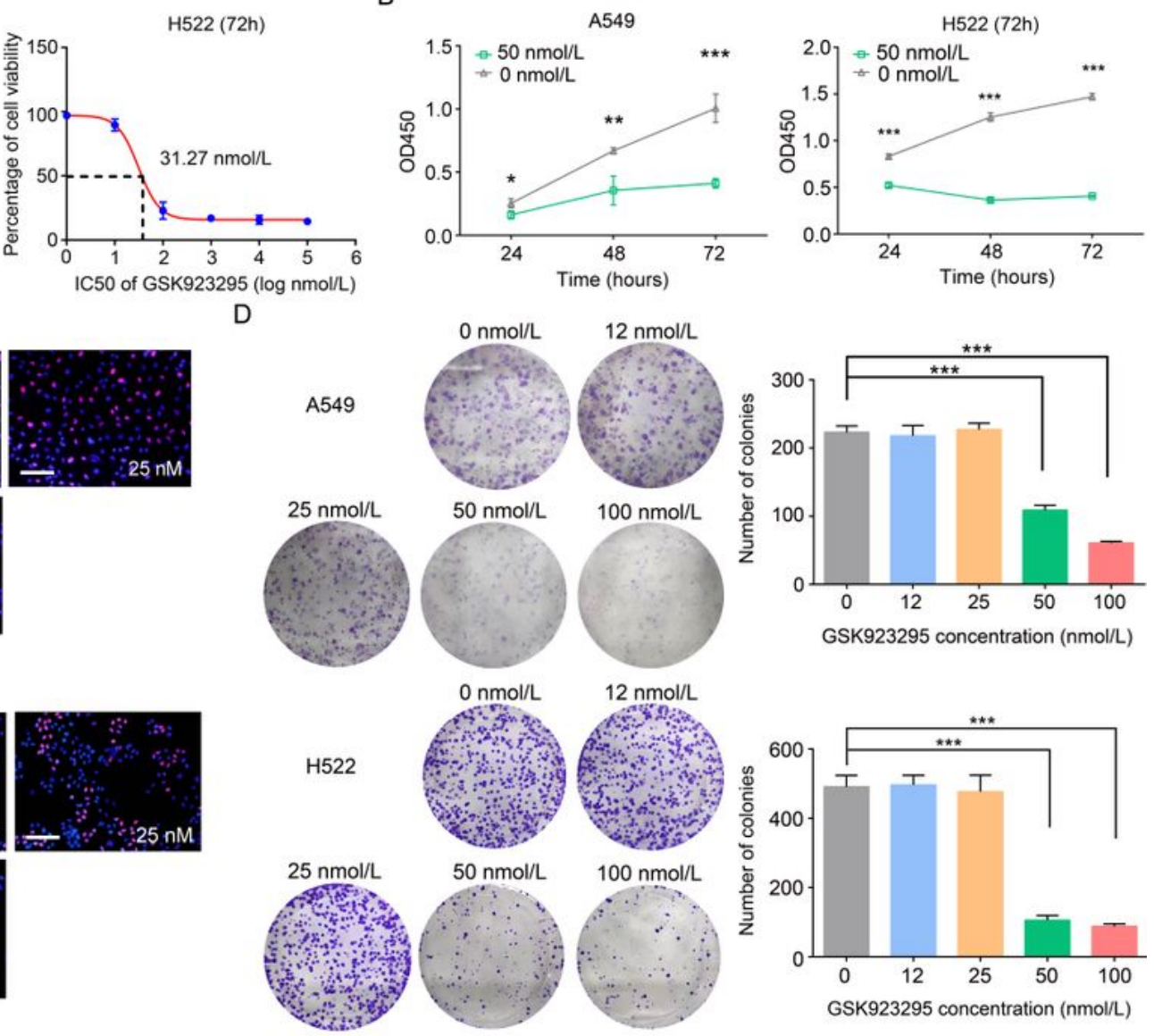

H522
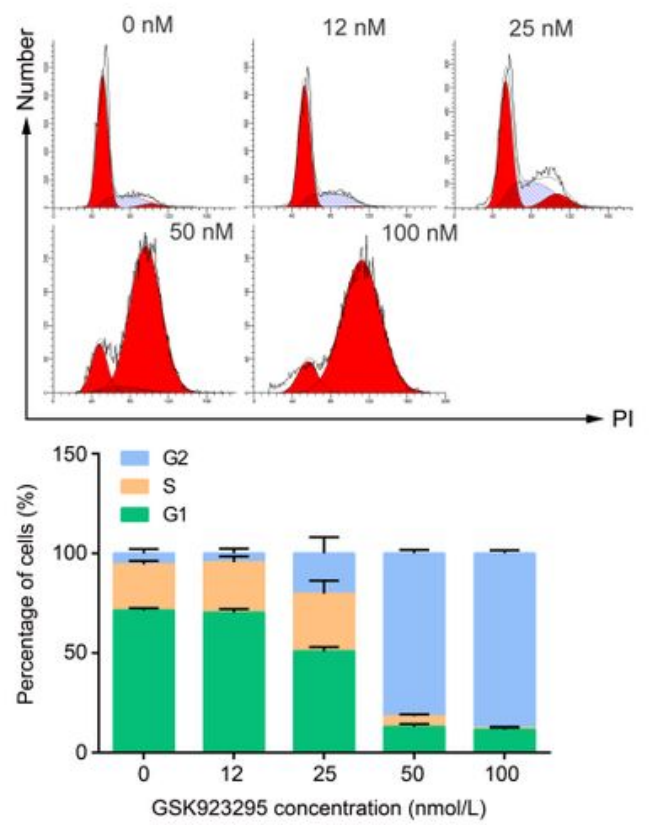

\section{Figure 1}

The role of a CENP-E inhibitor in lung cancer cells. (A) The IC50 of the CENP-E inhibitor GSK923295 in A549 and H522cells at $72 \mathrm{~h}$. (B-C) The proliferation activity of A549 and H522 cells after treatment with GSK923295. (D) The colony formation activity of A549 and H522 cells after treatment with GSK923295. (E) The cell cycle distribution of A549 and H522 cells after treatment with GSK923295. Values are the means \pm SD of three replicates. ${ }^{*} p<0.05$, ${ }^{* *} p<0.01$, ${ }^{* * *} p<0.001$ compared to the control. 
A

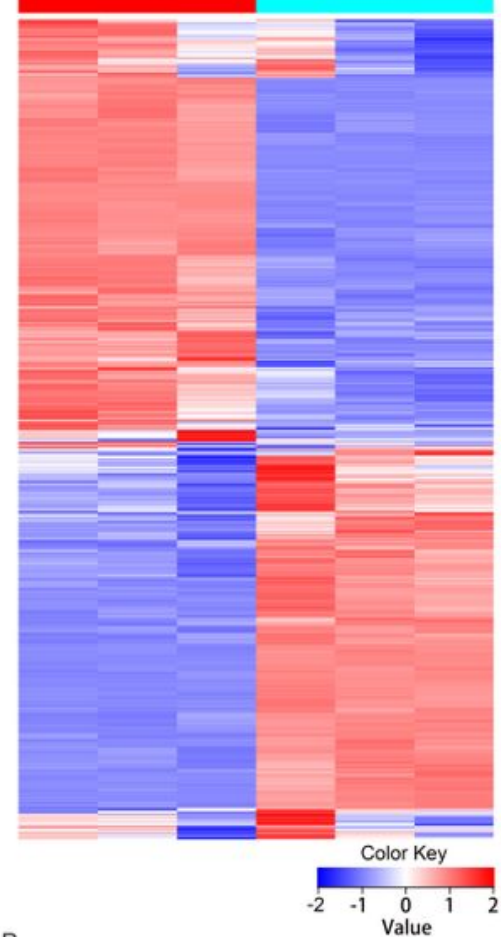

B

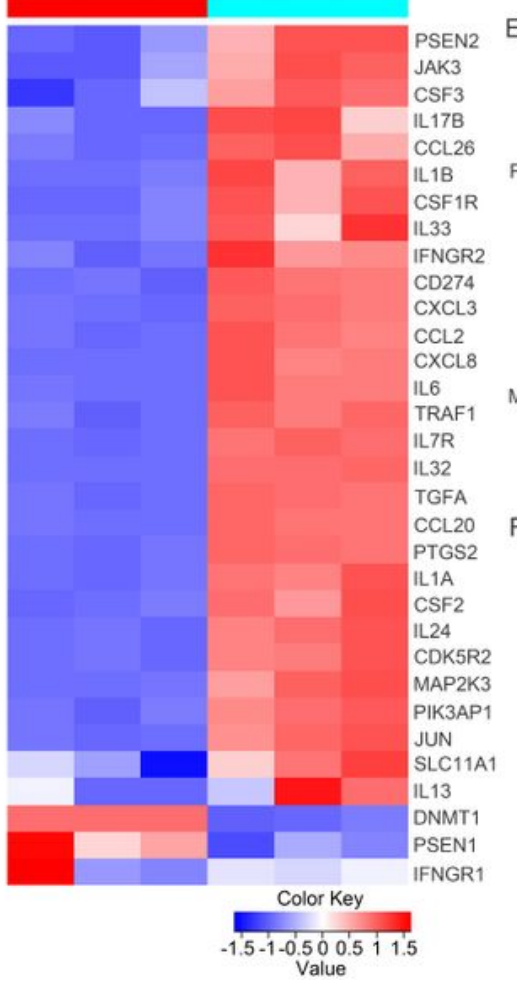

A549 24h

A549 $48 \mathrm{~h}$ \begin{tabular}{ccc} 
GSK923295 concentration (nmol/L) \\
\hline $12 \quad 25 \quad 50 \quad 100$
\end{tabular}

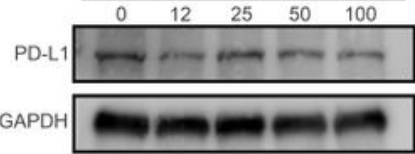

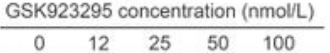
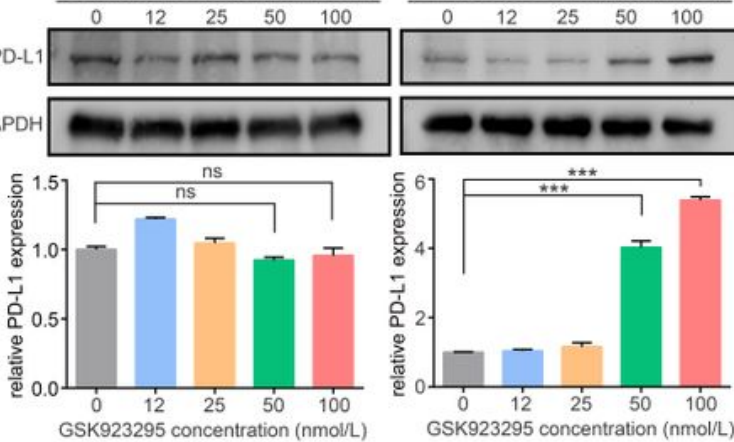

H522 24h

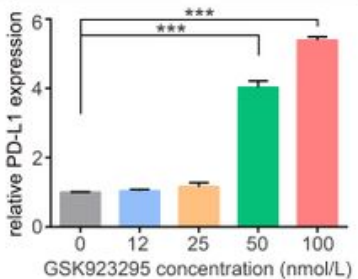

H522 48h GSK923295 concentration ( $\mathrm{nmol} / \mathrm{L}$ ) GSK923295 concentration ( $\mathrm{nmol} / \mathrm{L}$ )

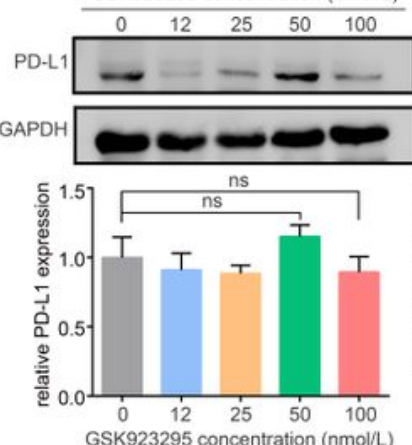

$E$
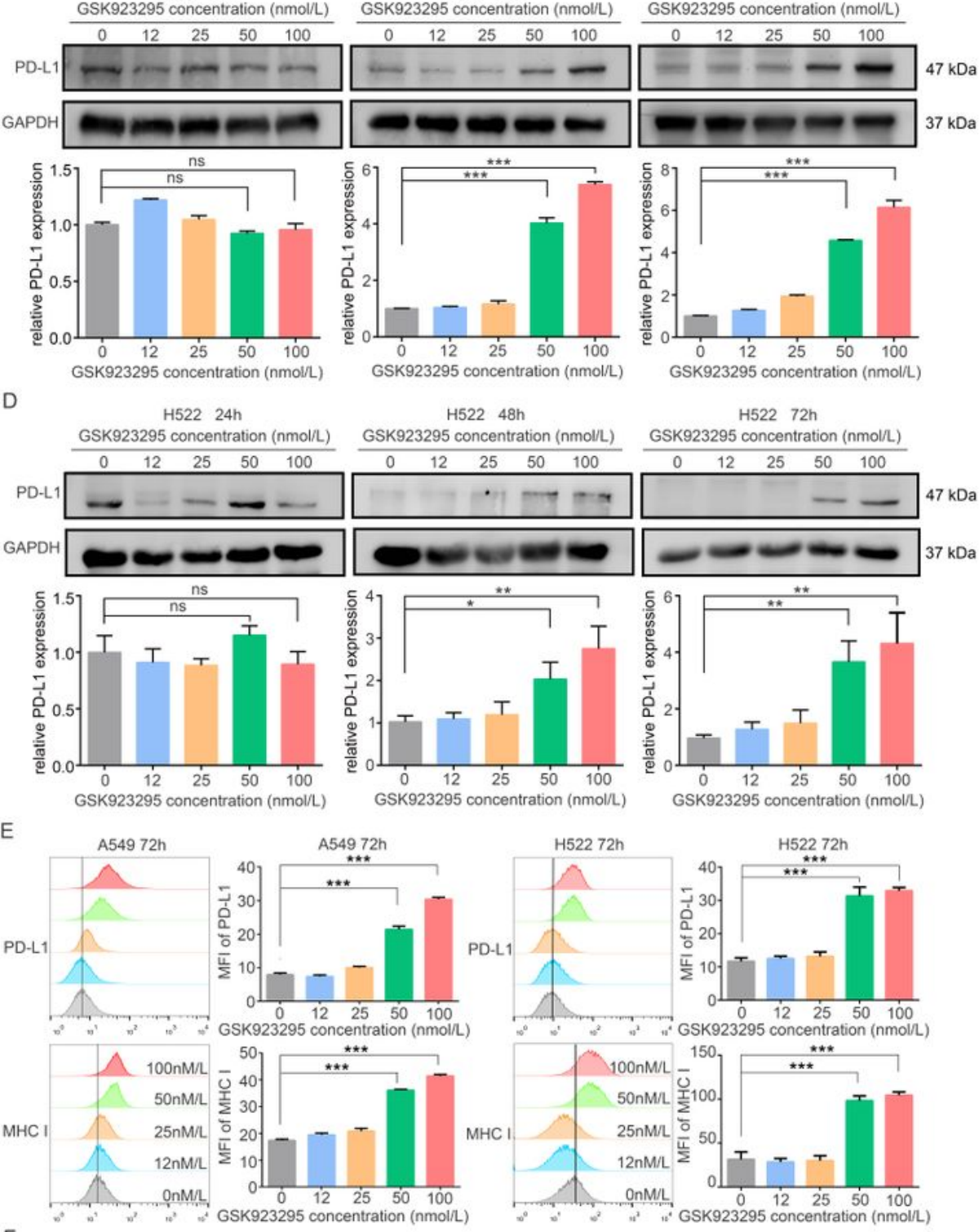

H522 $72 \mathrm{~h}$

GSK923295 concentration ( $\mathrm{nmol} / \mathrm{L}$ )
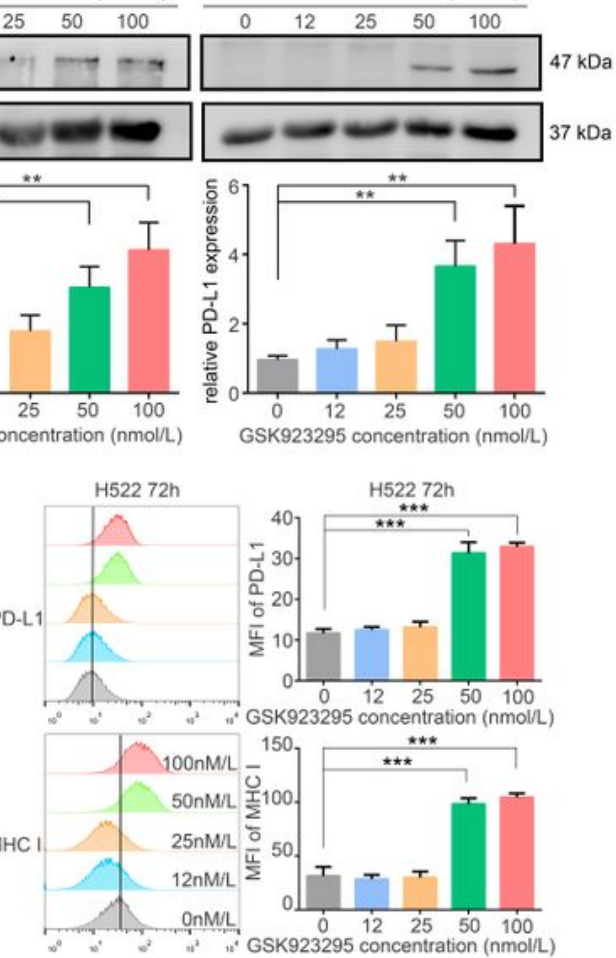
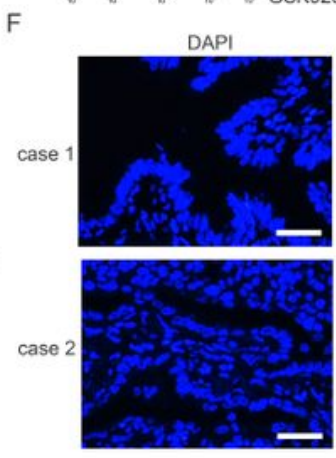
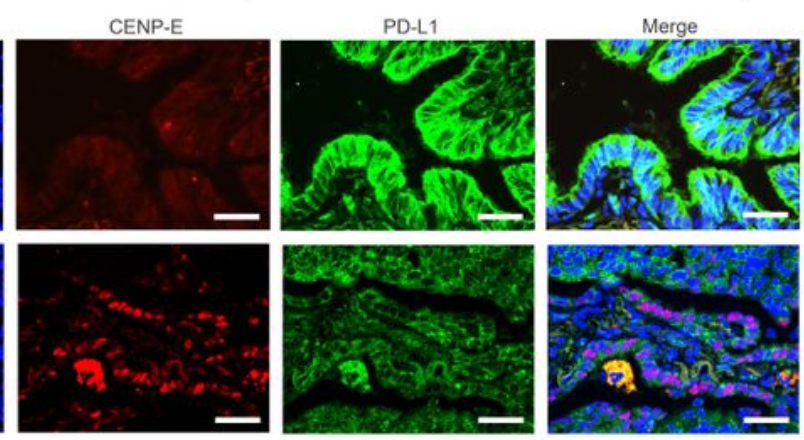

\section{Figure 2}

The inhibition of CENP-E induced the expression of PD-L1. (A) The changes in A549 cell mRNA caused by CENP-E inhibition with 100 nM/L GSK923295 (n=3). (B) The expression of immune molecules and genes regulating PD-L1 in A549 cells treated with 100 nM GSK923295 ( $n=3)$. The RNA sequencing heat-maps in (A) and (B) show the data from three individual experiments. (C) qPCR and WB analyses of PD-L1 expression in A549 cells treated with 0, 12, 25, 50 and $100 \mathrm{nM} / \mathrm{L}$ GSK923295 (n=3). (D) qPCR and WB 
analyses of PD-L1 expression in H522 cells after inhibition of CENP-E (n=3). Data in (C) and (D) are shown as the means \pm SD of three individual experiments. $(\mathrm{E})$ The mean fluorescence intensity (MFI) of PD-L1 and MHC I in A549 and H522 cells treated with GSK923295 using FACS analyses (n=3). Data are shown as the means \pm SD of biological triplicates. $(F)$ The expression of CENP-E and PD-L1 in human lung tumor tissues $(n=4)$. (Unpaired, two-tailed Student's $t$ test. ns=not significant, ${ }^{*} p<0.05,{ }^{*} p<0.01$, $\star \star \star ~ p<0.001$.
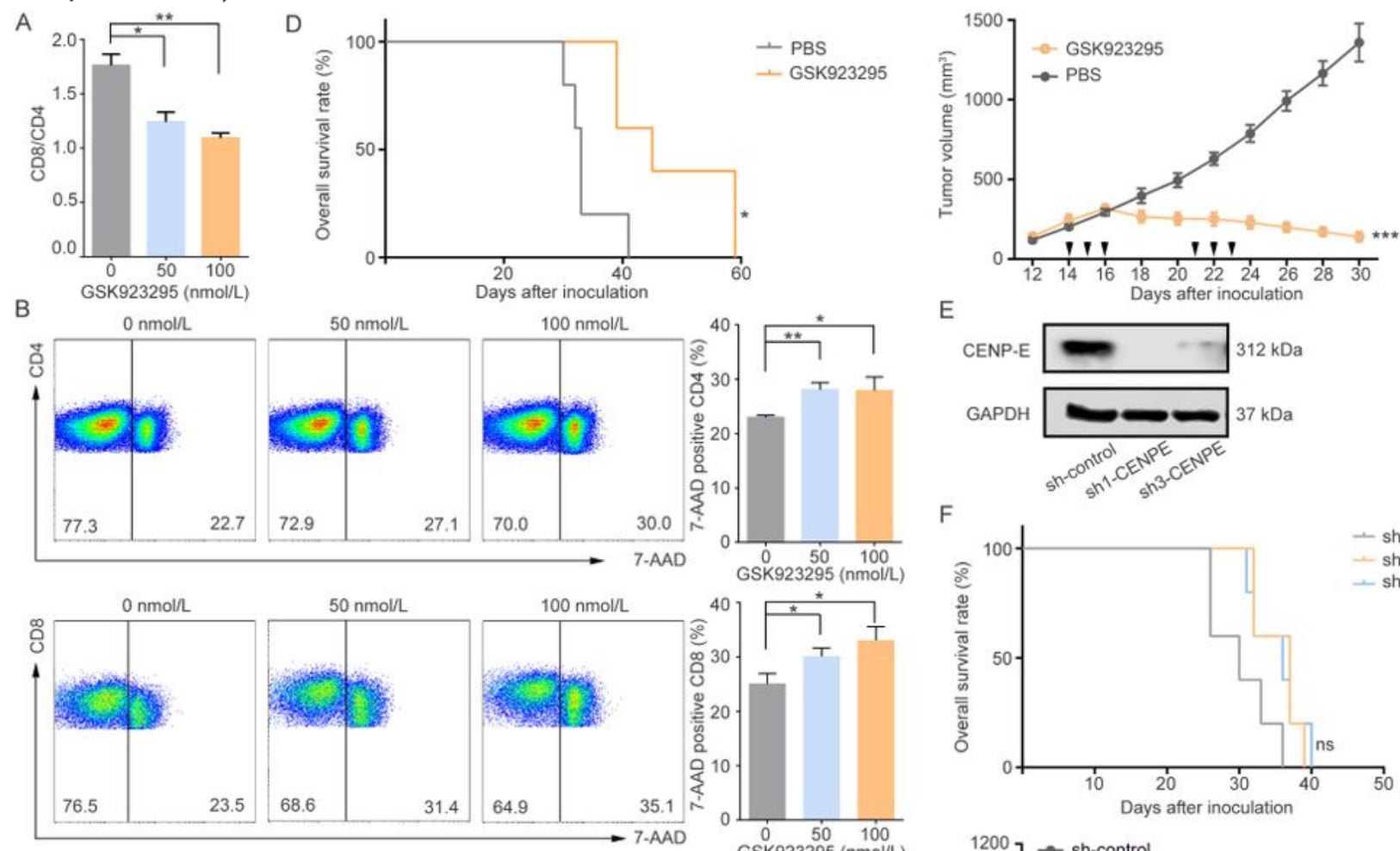

F
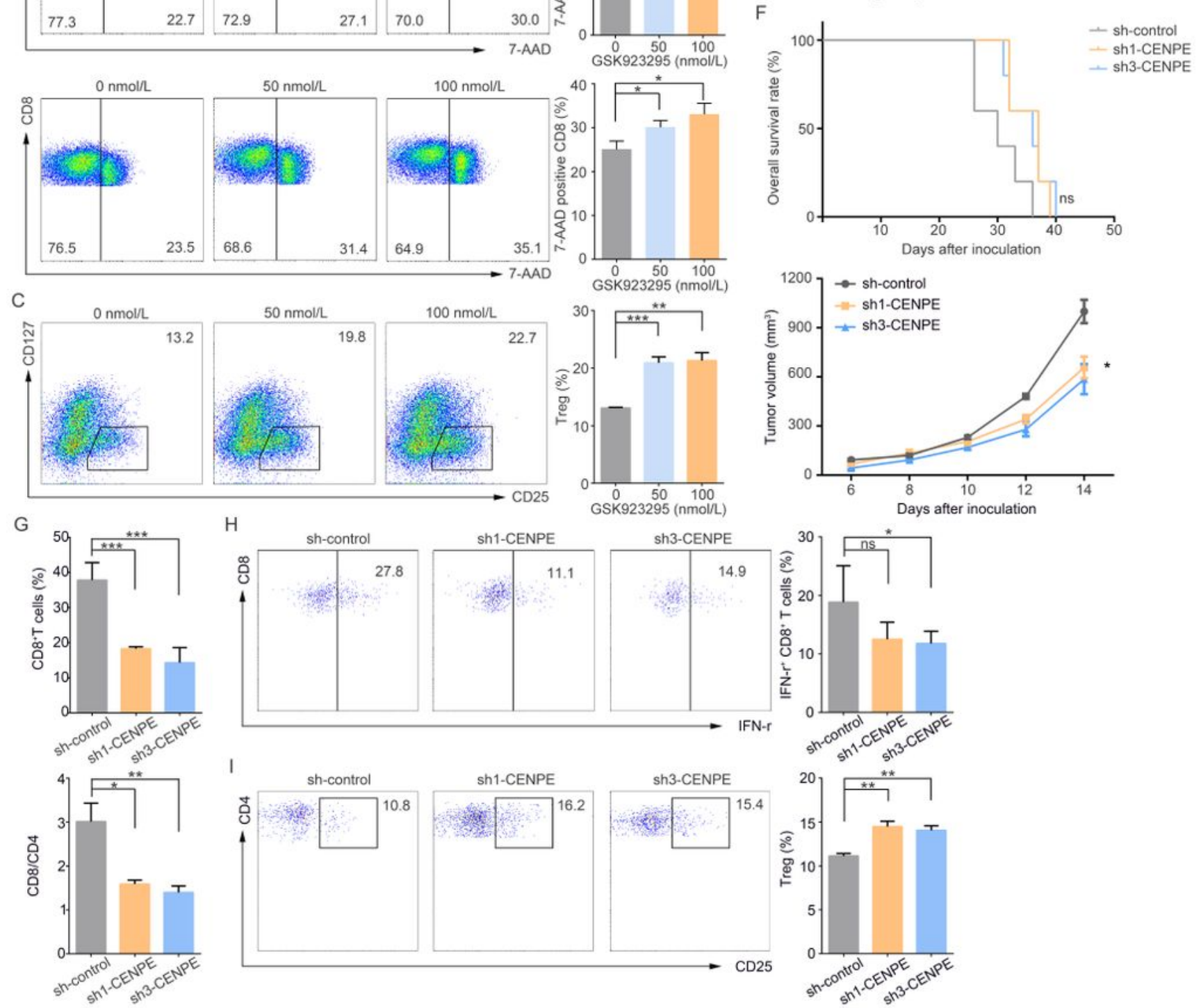

Figure 3 
CENP-E signal inhibition induces T cell suppression in vitro and in vivo. (A) Changes in the ratio of CD8+ $T$ cells to $C D 4+T$ cells in a coculture system of immune cells isolated from malignant pleural effusion (MPE) and A549 cells treated with $50 \mathrm{nM} / \mathrm{L}$ and $100 \mathrm{nM} / \mathrm{L}$ GSK923295 ( $\mathrm{n}=3$ ). (B) Apoptosis of CD8+ T cells and CD4+ T cells in a coculture system of immune cells and A549 cells treated with GSK923295 $(n=3)$. (C) Changes in the proportion of Tregs in the coculture system of immune cells and A549 cells treated with GSK923295 $(n=3)$. Data in (A)-(C) are shown as the means \pm SD of biological triplicates. (D) The tumor growth and survival curves for A549 cells subcutaneously transplanted into nude mice via intraperitoneal injection with PBS or GSK923295 (125 mg/kg, each injection is marked with arrows) ( $\mathrm{n}=5$ per group). Means \pm SD. (E) The efficacy of CENP-E knockdown verified using Western blotting $(n=3)$. Means \pm SD of biological triplicates. $(F)$ The tumor growth and survival curves for control and CENP-E knockdown Lewis cells subcutaneously transplanted into C57BL/ 6 mice ( $n=5$ per group). Means \pm SD. (G)-(I) FACS analysis of CD8+ T cells. (G) IFNY+T cells and Tregs in the Lewis C57BL/6 mice model $(n=5$ per group). Means $\pm S D$. (Unpaired, two-tailed Student's $t$ test. Ns=not significant, * means $p<0.05$, ** means $p<0.01$, *** means $p<0.001$.)
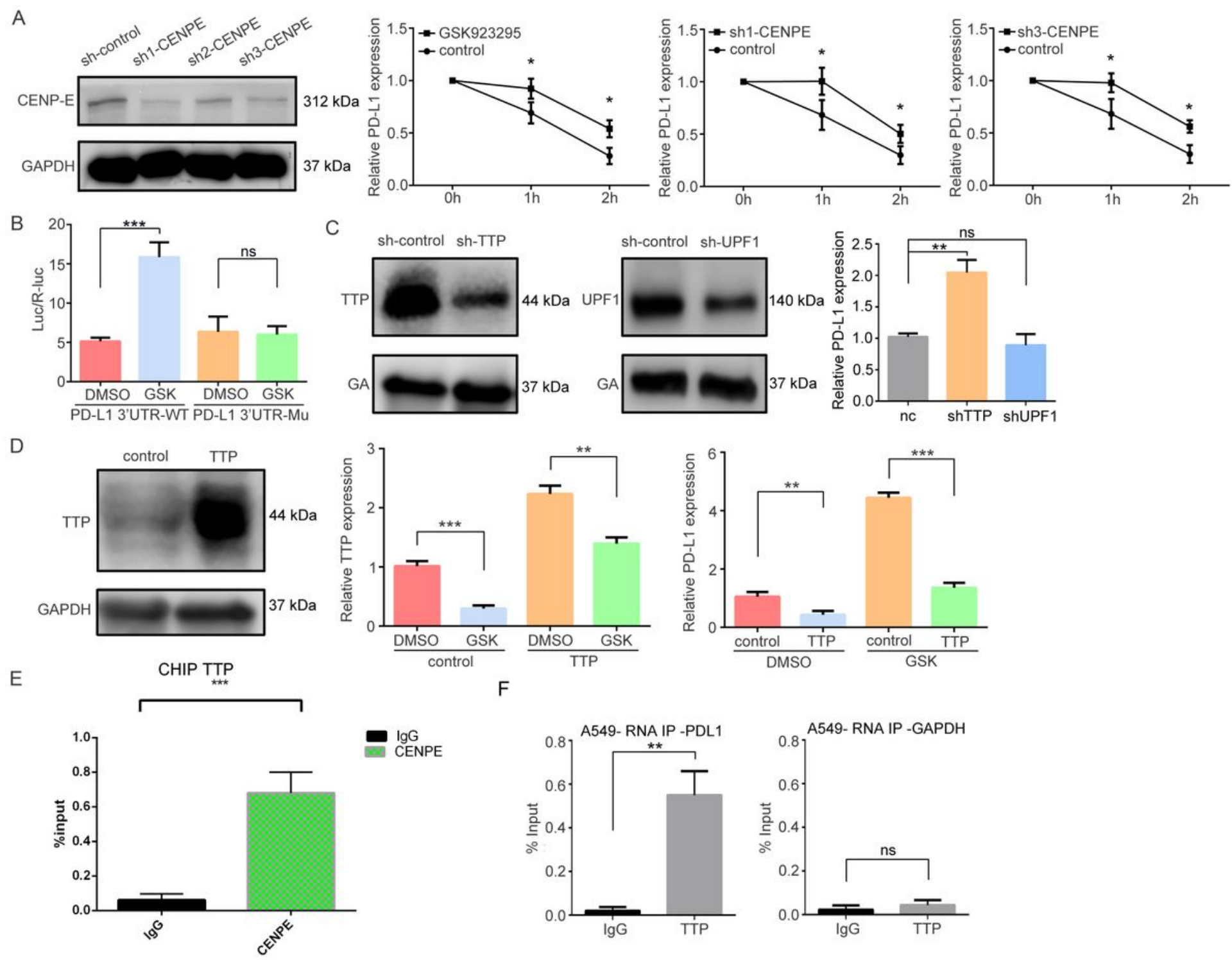

F

\section{Figure 4}


The inhibition of CENP-E-stabilized PD-L1 mRNA by TTP targeting of the 3'UTR. (A)The efficacy of CENPE knockdown verified using Western blotting (left), qPCR analysis of PD-L1 mRNA stability after the addition of actinomycin D (ACTD $5 \mu \mathrm{M} / \mathrm{L})$ in A549 cells treated with or without GSK923295 $(100 \mathrm{nM} / \mathrm{L})$ $(n=3)$. Data are shown as the means \pm SD of biological triplicates. (B) Normalized luciferase activity in A549 cells from wild-type or mutant PD-L1 3'UTR reporters, $48 \mathrm{~h}$ after treatment $(n=3)$. (C) The efficacy of TTP or UPF1 knockdown verified using Western blotting (left), qPCR analysis of PD-L1 mRNA expression in A549 cells after the knockdown of TTP and UPF1 $(n=3)$. (D) The efficacy of TTP over-expression verified using Western blotting (left), The mRNA expression of TTP after treatment with GSK923295 (100 $n M / L)$ and PD-L1 mRNA expression after the overexpression of TTP $(n=3)$. (E) CENPE ChIP DNA from the A549 cells were subjected to qPCR to validate the same binding regions of several target TTP genes. $(F)$ qPCR analysis of the RNA- immunoprecipitates from A549 cells $(n=3)$. Data in (A)-(F) are shown as the means \pm SD of biological triplicates. Unpaired, two-tailed Student's $t$ test. (ns=not significant, ${ }^{*} p<0.05$, ${ }^{* \star} p<0.01, * \star * p<0.001$.) 

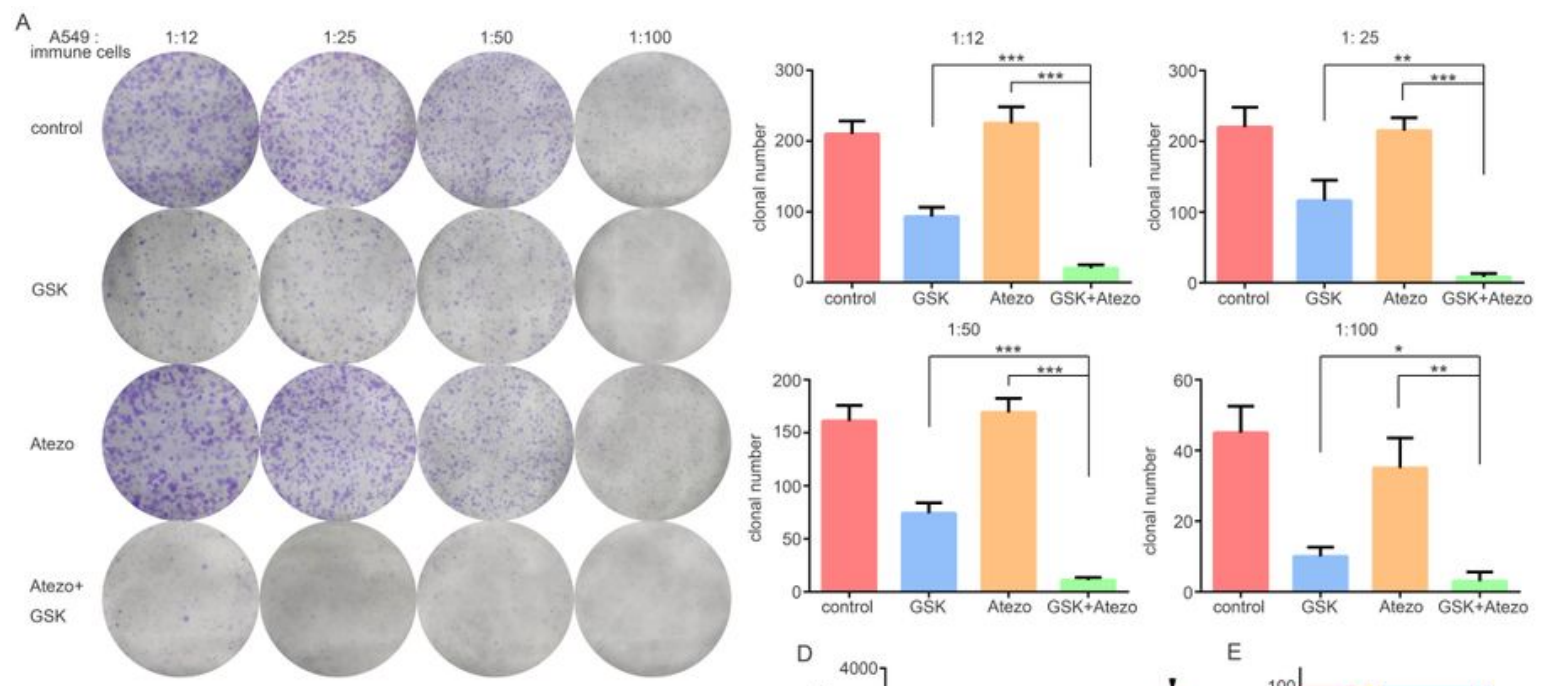

B

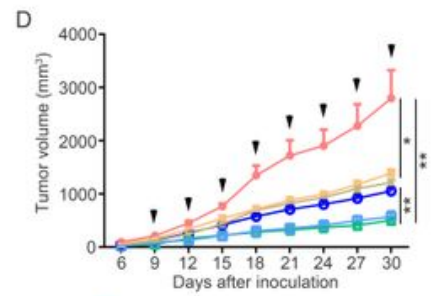

E
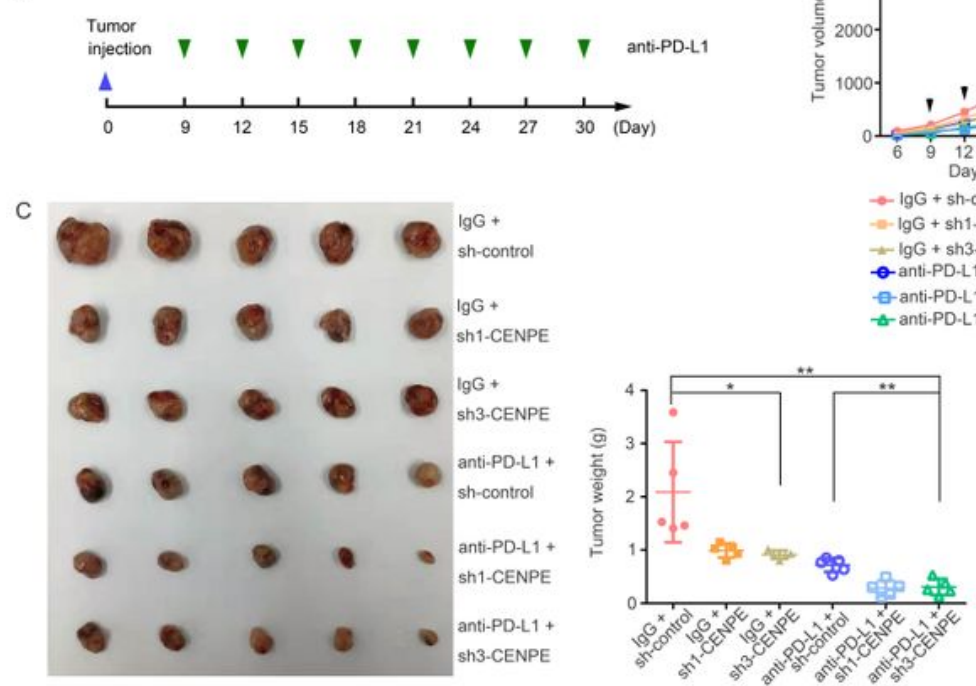

$\mathrm{F}$
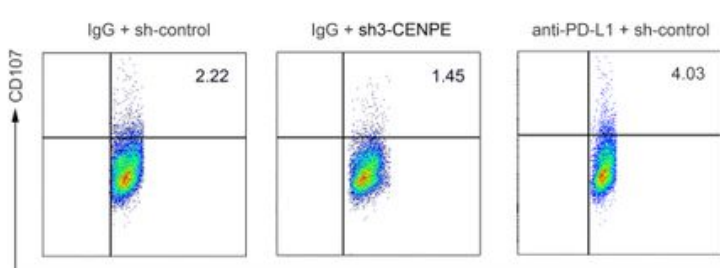

anti-PDL $1+$ sh3-CENPE
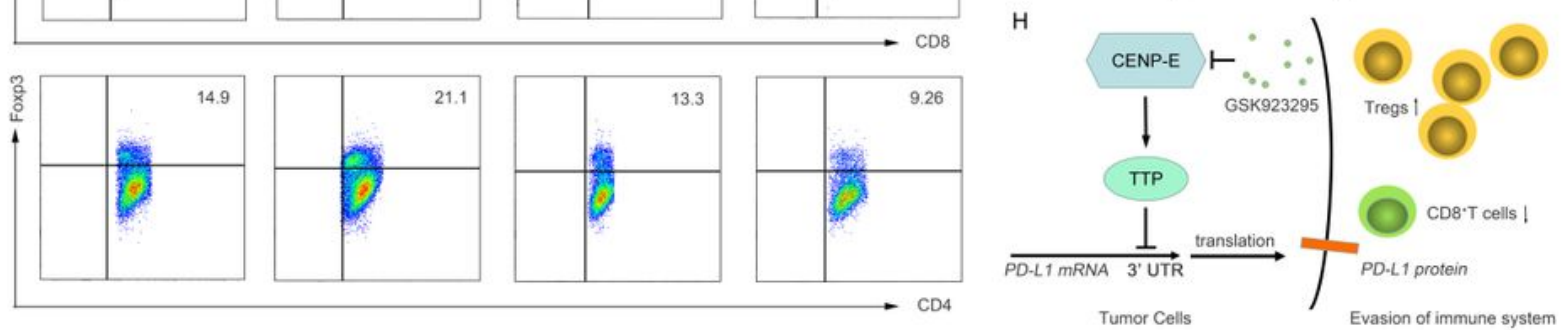

Figure 5

CENP-E inhibition combined with anti-PD-L1 antibodies enhanced the antitumor effect. (A) The colony numbers of A549 cells treated with the CENP-E inhibitor GSK923295 (50 nM/L) and anti-PD-L1 antibody $(10 \mu \mathrm{g} / \mathrm{ml})$ in a coculture system of A549 and immune cells isolated from MPE at ratios of 1:12, 1:25, 1:50 and 1:100 $(n=3)$. Data are shown as the means \pm SD of biological triplicates. (B) Schematic of drug intervention protocol for the PDL1 antibody in C57BL/ 6 mice, the C57BL/6 mice subcutaneously 
transplanted with control- or CENP-E knockdown- Lewis cells were then intraperitoneally injected with control IgG or anti-PD-L1 antibody (200 $\mu$ g per mice, each injection is marked with arrows) . (C) The tumor weight at 30 days after the mice in (B) were sacrificed ( $n=5$ per group). Means $\pm S D$. (D)The tumor growth curves for. Means $\pm S D$. (D-E) The survival curves for the Lewis lung tumor mice models established and treated using the same method described in (B) ( $n=5$ per group). (F-G) Tumor infiltration of CD107/CD8 T cells and Foxp3/CD4 T cells in conshCENP-E group, shCENP-E group, conshCENP-E+

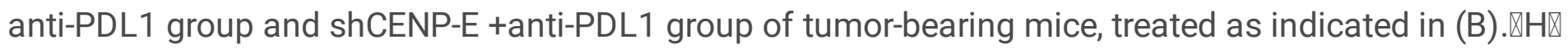
The model of the inhibition CENPE signal and immune response. Means \pm SD. (Unpaired, two-tailed Student's t test. ${ }^{*} p<0.05, * \star p<0.01, \star \star \star p<0.001$.) 

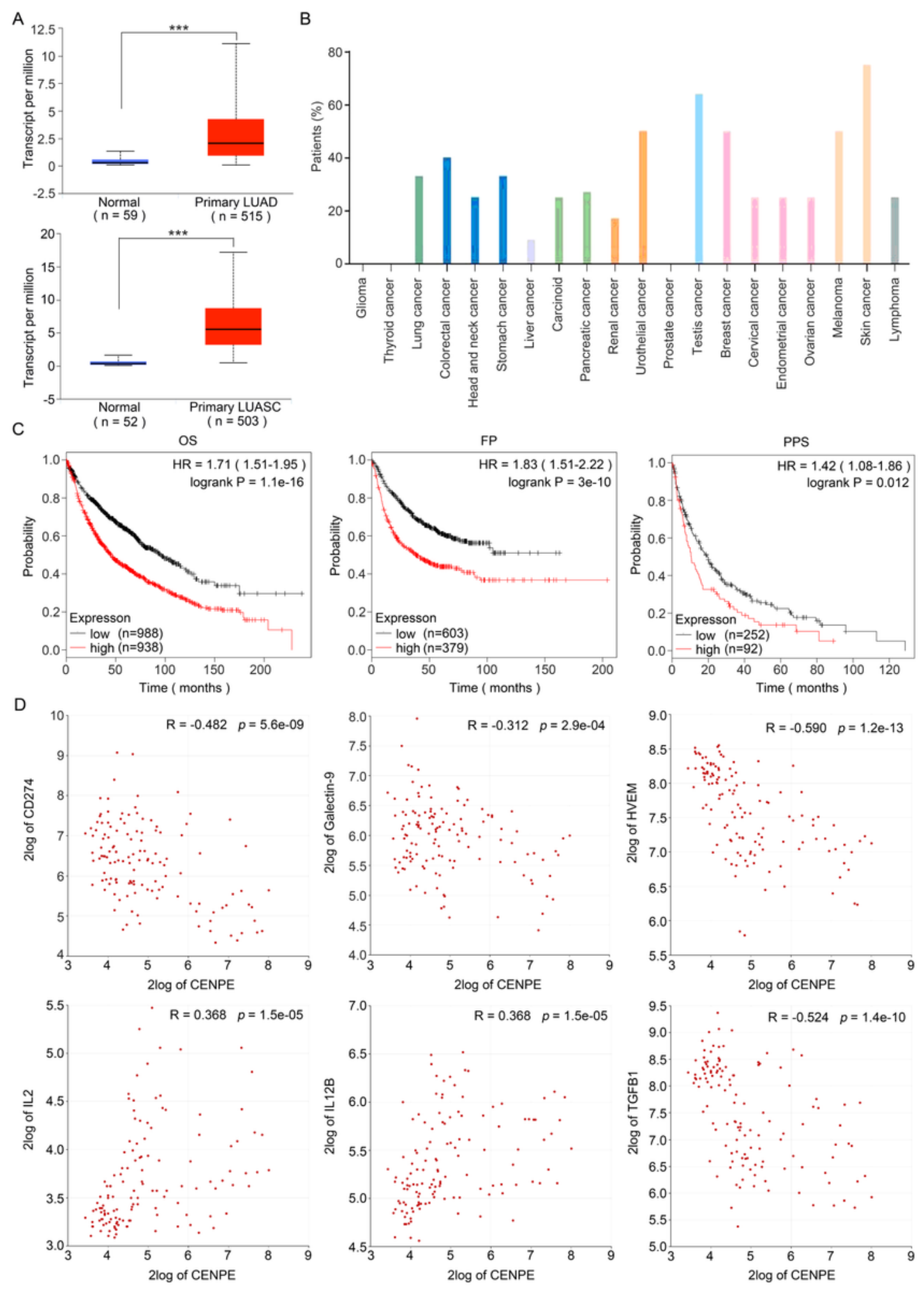

\section{Figure 6}

The expression and the correlation between CENP-E and clinical outcome and the expression of immune molecules in lung cancer. (A) The expression of CENP-E in lung tumor tissues on UALCAN. (B) The expression of CENP-E in lung cancer and other cancers (data from The Human Protein Atlas). (C) The prognostic value of CENP-E in lung cancer patients from the website Kaplan-Meier Plotter. (D) The relationship between the expression of CENP-E and immune molecules, including checkpoint molecules 
PD-L1 (CD274), galectin-9 and HVEM (the upper row), and cytokines IL2, IL12B, TGFB1 (the lower row). $(\star \star \star ~ p<0.001$.)

\section{Supplementary Files}

This is a list of supplementary files associated with this preprint. Click to download.

- Supplementalmethods.docx 